\title{
Stem cell factor stimulates neurogenesis in vitro and in vivo
}

\author{
Kunlin Jin, Xiao Ou Mao, Yunjuan Sun, Lin Xie, and David A. Greenberg \\ Buck Institute for Age Research, Novato, California, USA
}

Cerebral ischemia stimulates neurogenesis in proliferative zones of the rodent forebrain. To identify the signaling factors involved, cerebral cortical cultures prepared from embryonic mouse brains were deprived of oxygen. Hypoxia increased bromodeoxyuridine (BrdU) incorporation into cells that expressed proliferation markers and immature neuronal markers and that lacked evidence of DNA damage or caspase-3 activation. Hypoxia-conditioned medium and stem cell factor (SCF), which was present in hypoxia-conditioned medium at increased levels, also stimulated BrdU incorporation into normoxic cultures. The SCF receptor, c-kit, was expressed in neuronal cultures and in neuroproliferative zones of the adult rat brain, and in vivo administration of SCF increased BrdU labeling of immature neurons in these regions. Cerebral hypoxia and ischemia may stimulate neurogenesis through trophic factors, including SCF.

J. Clin. Invest. 110:311-319 (2002). doi:10.1172/JCI200215251.

\section{Introduction}

The adult vertebrate brain retains the capacity for neurogenesis, which resides largely in selected regions that harbor neuronal precursor cells throughout life. These include the subventricular zone (SVZ), especially that portion adjacent to the most rostral parts of the lateral ventricles (1-3), and the subgranular zone (SGZ) of the hippocampal dentate gyrus (DG) (4). Some reports suggest that additional regions, such as the cerebral neocortex, may also generate new neurons in the adult, but this is disputed (5).

Understanding the signals that trigger neuronal proliferation in the brain in vivo could assist the development of cell-replacement therapy for neurological disorders such as stroke. Efforts to identify these signals have been aided by the ability to grow neuronal precursor cells in vitro. Several factors can stimulate neurogenesis in such systems, including EGF (6), FGF-2 (7), and brain-derived neurotrophic factor (BDNF) (1). In addition, some studies have shown that cultured progenitor cells (8-10) or tissue explants containing axons that project to neuroproliferative zones (11) release factors

Received for publication February 11, 2002, and accepted in revised form June 10, 2002.

Address correspondence to: David A. Greenberg, Buck Institute for Age Research, 8001 Redwood Boulevard, Novato, California 94945, USA. Phone: (415) 209-2087; Fax: (415) 209-2230;

E-mail: dgreenberg@buckinstitute.org.

Conflict of interest: No conflict of interest has been declared. Nonstandard abbreviations used: subventricular zone (SVZ); subgranular zone (SGZ); dentate gyrus (DG); brain-derived neurotrophic factor (BDNF); middle cerebral artery (MCA); bromodeoxyuridine (BrdU); proliferating cell nuclear antigen (PCNA); stem cell factor (SCF); DNA polymerase I-mediated biotin-dATP nick translation (PANT); neuronal nuclear antigen (NeuN); microtubule-associated protein-2 (MAP-2); glial fibrillary acidic protein (GFAP); embryonic nerve cell adhesion molecule (E-NCAM); 4',6-diamidine-2-phenylindole dihydrochloride (DAPI); humanized Renilla reniformis green fluorescent protein (hrGFP); 3-(4,5-dimethylthiazol-2-yl)-2,5diphenyl tetrazolium bromide (MTT); artificial cerebrospinal fluid (aCSF); hypoxia-conditioned medium (HCM). into conditioned medium that can regulate neurogenesis. In some cases, administration or overexpression of neurotrophic factors can enhance neurogenesis in neuroproliferative zones of the adult brain in vivo (12-15).

In addition to its role in development, neurogenesis also occurs in response to cerebral injury, including excitotoxic damage $(16,17)$, seizures $(18)$, and oxidative stress-induced apoptosis (19). We have focused on the ability of cerebral ischemia to stimulate neurogenesis because of its potential implications for stroke recovery and treatment. In a previous study (20), we found that focal ischemia caused by occlusion of the middle cerebral artery (MCA) in the rat for 90 minutes increased the incorporation of BrdU in the SGZ and SVZ bilaterally. Cells labeled with bromodeoxyuridine (BrdU) coexpressed proliferating cell nuclear antigen (PCNA) and the immature neuronal marker doublecortin. Other reports indicate that global cerebral ischemia triggers neurogenesis in the SGZ $(21,22)$, that focal cerebral ischemia induces neurogenesis in peri-infarction cortex $(23,24)$, and that FGF-2 may be responsible for the proliferation and differentiation of neuronal progenitor cells in DG after focal ischemia (17). However, the biological basis of hypoxia- or ischemia-induced neurogenesis remains poorly understood.

To address this issue, we used embryonic mouse cerebral cortical cultures enriched in cells of neuronal lineage and deprived of oxygen to simulate ischemia (25). The embryonic age at which cultures are prepared (embryonic day 16-17 [E16-17]) corresponds to a period of active cortical neurogenesis (26). Our results indicate that hypoxia induces neurogenesis in mouse cortical cultures and that this effect is mediated by secreted factors such as stem cell factor (SCF), which stimulates neurogenesis in cortical cultures and in SVZ and SGZ in vivo.

\section{Methods}

Cell culture and in vitro bypoxia. Cerebral cortical cultures were prepared from 16-day Charles River CD1 mouse 
embryos as described (27), except that Neurobasal medium containing $2 \% \mathrm{~B}_{27}$ supplement, $2 \mathrm{mM}$ glutamate, and $1 \%$ penicillin and streptomycin (Life Technologies Inc., Rockville, Maryland, USA) was used (28). After 4 days, one-half of the medium was replaced with Neurobasal medium containing $2 \% \mathrm{~B}_{27}$, and experiments were conducted at 6-7 days. Cultures were placed in modular incubator chambers (Billups-Rothenberg, Del Mar, California, USA) for $0-24$ hours at $37^{\circ} \mathrm{C}$ in humidified $95 \%$ air $/ 5 \% \mathrm{CO}_{2}$ (control) or humidified $95 \%$ $\mathrm{N}_{2} / 5 \% \mathrm{CO}_{2}$ (hypoxia), then returned to normoxic conditions for the remainder, if any, of the 24 hours (25). Focal cerebral ischemia. Focal ischemia was induced in 280 - to 300 -g adult male Sprague-Dawley rats by intraluminal occlusion of the MCA with a suture as previously described $(20,29)$. The suture was left in place for 90 minutes and then withdrawn, and rats were sacrificed 1 week later.

BrdU labeling. BrdU $(50 \mu \mathrm{g} / \mathrm{ml}$; Sigma-Aldrich, St. Louis, Missouri, USA) was added to cultures 20 minutes prior to the onset of exposure to hypoxia for 0 to 24 hours, and cultures were processed for immunostaining as described (30). For in vivo studies, BrdU was administered intraperitoneally, as reported previously (20).

BrdU-immunopositive cell counts. BrdU-positive cells in culture were counted in five fields per well (center and at 3, 6, 9, and 12 o'clock). In brain sections, BrdU-positive cells were counted in five to seven $50-\mu \mathrm{m}$ coronal sections per animal, spaced $200 \mu \mathrm{m}$ apart, by a researcher blinded to the experimental conditions, using a Nikon E300 epifluorescence microscope equipped with a Magnifire digital color camera (ChipCoolers Inc., Warwick, Rhode Island, USA). Cells containing densely brown-stained nuclei with clear morphology were considered BrdU positive. Results were expressed as BrdU-positive cells per field or section.

Assays of DNA strand breaks. DNA single- and doublestrand breaks were detected by DNA polymerase I-mediated biotin-dATP nick translation (PANT) labeling, Klenow labeling, or TUNEL, as described previously $(31,32)$.

Immunocytochemistry. Cell cultures (30) and brain sections (20) were processed for immunocytochemistry as described previously. Primary Ab's were: mouse monoclonal anti-BrdU $(2 \mu \mathrm{g} / \mathrm{ml}$, Roche, Indianapolis, Indiana, USA); sheep polyclonal anti-BrdU $(25 \mu \mathrm{g} / \mathrm{ml}$; BIODESIGN International, Saco, Maine, USA); mouse monoclonal anti-neuronal nuclear antigen (1:200; anti-NeuN), mouse monoclonal anti-PCNA (1:100), mouse monoclonal anti-CD11b (1:50), mouse monoclonal antiCD146 (1:50), goat polyclonal anti-vimentin (1:40), and rabbit polyclonal anti-SCF (1:100) (Chemicon International, Temecula, California, USA); mouse monoclonal anti-microtubule-associated protein-2 (1:100; antiMAP-2), mouse monoclonal anti-glial fibrillary acidic protein (1:400; GFAP), and rabbit polyclonal antifibronectin (1:400) (Sigma-Aldrich); rabbit polyclonal anti-phospho-histone H3 (1:150; Upstate Biotechnology Inc., Lake Placid, New York, USA); rat monoclonal anti-embryonic nerve cell adhesion molecule (1:100; anti-E-NCAM) (BD Transduction Laboratories, San Diego, California, USA); mouse monoclonal anti-CDC47 (1:100; Lab Vision Corporation, Fremont, California, USA); affinity-purified goat polyclonal anti-Neuro D (1:100; Santa Cruz Biotechnology Inc., Santa Cruz, California, USA); mouse monoclonal anti-nestin (1:400) and, anti-c-kit (1:100) (BD PharMingen, San Diego, California, USA); and rabbit polyclonal $\mathrm{Ab}$ against cleaved (17-20 kDa) caspase-3 (1:250; New England Biolabs Inc., Beverly, Massachusetts, USA). Secondary Ab's were: FITC-conjugated goat anti-mouse IgG, FITC-conjugated rabbit anti-rat IgG, and FITC-conjugated rabbit antirabbit IgG (1:200; Vector Laboratories, Burlingame, California, USA), and FITC-conjugated pig anti-goat $\operatorname{IgG}$, rhodamine-conjugated rat-absorbed donkey anti-mouse IgG, rhodamine-conjugated rat-absorbed donkey antirabbit IgG, and rhodamine-conjugated rat-absorbed donkey anti-sheep IgG (1:200; Jackson ImmunoResearch Laboratories Inc., West Grove, Pennsylvania, USA). DAPI (4',6-diamidine-2-phenylindole dihydrochloride; Vector Laboratories) was used to counterstain nuclei, and fluorescence signals were detected as described (20). Controls included omitting or preabsorbing the primary or omitting the secondary $\mathrm{Ab}$.

Retroviral infection. Humanized Renilla reniformis green fluorescent protein (hrGFP) was cloned downstream of the viral promoter of the $\mathrm{pFB}$ retroviral vector (Stratagene, La Jolla, California, USA). The vector was produced by transiently transfecting NIH $3 \mathrm{~T} 3$ cells with two additional vectors expressing gag-pol and vesicular stomatitis virus $\mathrm{G}$ envelope protein. The $\mathrm{pFB}-\mathrm{hrGFP}$ supernatant containing $4.9 \times 10^{7}$ or greater infectious virus particles was filtered through a $0.45-\mu \mathrm{m}$ filter and frozen at $-80^{\circ} \mathrm{C}$. Ten microliters of retroviral supernatant was added to each well of four-well plates containing neuron cultures in $500 \mu \mathrm{l}$ of medium, incubated at $37^{\circ} \mathrm{C}$ for 2 days, and then for 24 hours in normoxic or hypoxic conditions. GFP-expressing cells were detected with a Nikon E300 microscope with excitation at 470 and emission at $505 \mathrm{~nm}$.

Hypoxia-conditioned medium. Medium $(500 \mu \mathrm{l} /$ well $)$ was collected from normoxic or hypoxic cultures on four-well plates, pipetted into the sample reservoir of a Microcon YM-100 centrifugal filter device (Millipore Corp., Bedford, Massachusetts, USA) that had been spin rinsed with medium and centrifuged according to the manufacturer's instructions. Constituents with relative molecular mass $\left(\mathrm{M}_{\mathrm{r}}\right)$ greater than 100,000 were recovered by rinsing with $500 \mu \mathrm{l}$ of medium, and constituents with $M_{r}$ less than 100,000 were transferred to a YM-50 filter device and the same steps repeated. Successive fractions were collected by applying the filtrates to YM30, YM10, and YM3 filter devices. Fractions (500 $\mu \mathrm{l} /$ well of a four-well plate) were added immediately to normoxic cultures together with $\mathrm{BrdU}$, and in some cases pFB-hrGFP, for 24-72 hours.

Cell viability. Cell viability was assayed by incubating cells with 3-(4,5-dimethylthiazol-2-yl)-2,5-diphenyl tetra- 
zolium bromide (MTT) $(5 \mathrm{mg} / \mathrm{ml}$; Sigma-Aldrich) at $37^{\circ} \mathrm{C}$ for 2 hours. Medium was removed, and cells were solubilized with DMSO and transferred to 96-well plates. Absorbance at $570 \mathrm{~nm}$ was measured in a Cytofluor 4000 plate reader (PerSeptive Biosystems, Framingham, Massachusetts, USA). Results were expressed as a percentage of control absorbance measured in normoxic cultures after subtracting background absorbance measured in freeze-thawed cultures.

Western blotting. Blotting was performed as described previously (27); in some experiments, protein from 1.5-2.0 $\mathrm{ml}$ of culture supernatant was concentrated using a Microcon YM-3 centrifugal filter device (Millipore Corp.). The primary Ab's were: rabbit polyclonal anti-SCF, mouse monoclonal anti-EGF, rabbit polyclonal anti-TNF- $\alpha$ and rabbit polyclonal anti-FGF-2 (1:500; Chemicon International) and rat monoclonal anti- $\beta$-NGF (1:500, BD PharMingen). The secondary Ab's were: horseradish peroxidase-conjugated anti-mouse or anti-rat (for monoclonal primary) or anti-rabbit (for polyclonal primary) IgG (1:3,000; Santa Cruz Biotechnology Inc.). Differences in SCF protein expression on Western blots were quantified using a GS-710 calibrated imaging densitometer and Quantity One software (Bio-Rad Laboratories Inc., Hercules, Califoria, USA).

RT-PCR. Total RNA was isolated from cultured neurons using TRIzol reagent (Life Technologies Inc.) according to the manufacturer's instructions. RT-PCR was performed using the following primers: FGF-2 forward primer, 5'-AGAGCGACCCACACGTCAAAC-3' (160-181) and FGF-2 reverse primer, 5'-CCAACTGGAGTATTTCCGTGACC-3' (366-344) designed from mouse FGF-2 (GenBank accession number M30644) and SCF forward primer, 5 '-CGGGAATCCTGTGACTGATAATG-3' (236-258) and SCF reverse primer, 5'TGTCAGATGCCACCATAAAGTCC-3' (605-627) designed from mouse SCF (GenBank accession number M57647). Rat $\beta$-actin sense and antisense primers were used as controls for determining the quantity of RNA. PCR products were separated on $3 \%$ agarose gels using 100-bp ladder DNA standards as a size reference.

\section{Figure 1}

Hypoxia increases BrdU incorporation in cerebral cortical cultures. (a) Cortical cultures were stained with Ab's against the indicated markers and counterstained with the nuclear stain DAPI (blue). (b) The percentage of cells expressing each marker is shown, indicating that most cells at this stage in culture are immature neurons. (c) Cultures were treated with $\mathrm{BrdU}$ and deprived of oxygen for up to 24 hours, and BrdU was visualized by immunocytochemistry and quantified by cell counting. (d) The hypoxia-induced increase in BrdU incorporation was reduced by the cell cycle inhibitors aphidicolin, cytosine arabinoside, and hydroxyurea. Data shown are representative fields (a) or mean \pm SEM, $n=3$ (b-d).
Intraventricular administration of SCF. Adult male Sprague-Dawley rats were anesthetized with $4 \%$ isoflurane in $70 \% \mathrm{~N}_{2} \mathrm{O} / 30 \% \mathrm{O}_{2}$ and implanted with an osmotic minipump (Alzet 1003D; Alza Corporation, Mountain View, California, USA). The cannula was placed in the right lateral ventricle $4.0 \mathrm{~mm}$ deep to the pial surface, $+0.8 \mathrm{~mm}$ anteroposterior relative to bregma, and $1.3 \mathrm{~mm}$ lateral to the midline. Each rat was infused for 3 days with $1 \mu \mathrm{l} / \mathrm{h}$ of either human recombinant SCF $(1 \mu \mathrm{g} / \mathrm{ml}$; R\&D Systems Inc., Minneapolis, Minnesota, USA) in artificial cerebrospinal fluid (aCSF; $128 \mathrm{mM} \mathrm{NaCl}, 2.5 \mathrm{mM} \mathrm{KCl}, 0.95 \mathrm{mM} \mathrm{CaCl}_{2}, 1.99 \mathrm{mM}$ $\left.\mathrm{MgCl}_{2}\right)(n=6)$; SCF $(1 \mu \mathrm{g} / \mathrm{ml})$ plus anti-c-kit Ab $(10$ $\mu \mathrm{g} / \mathrm{ml})$ in aCSF $(n=4)$ or aCSF alone $(n=5)$. BrdU (50 $\mathrm{mg} / \mathrm{kg}$; Sigma-Aldrich) was dissolved in saline and given intraperitoneally, twice daily at 8-hour intervals, for 3 consecutive days, and rats were killed 1 week later. BrdU-positive cells were characterized by double-labeling with anti-NeuN and Neuro D Ab's as described (20). Confocal images were obtained with a Nikon PCN 2000 confocal microscope system.

Data analysis. Quantitative data were expressed as mean plus or minus SEM from at least three experiments. ANOVA and Student $t$ test were used for statistical analysis, with $P$ values less than 0.05 considered significant.

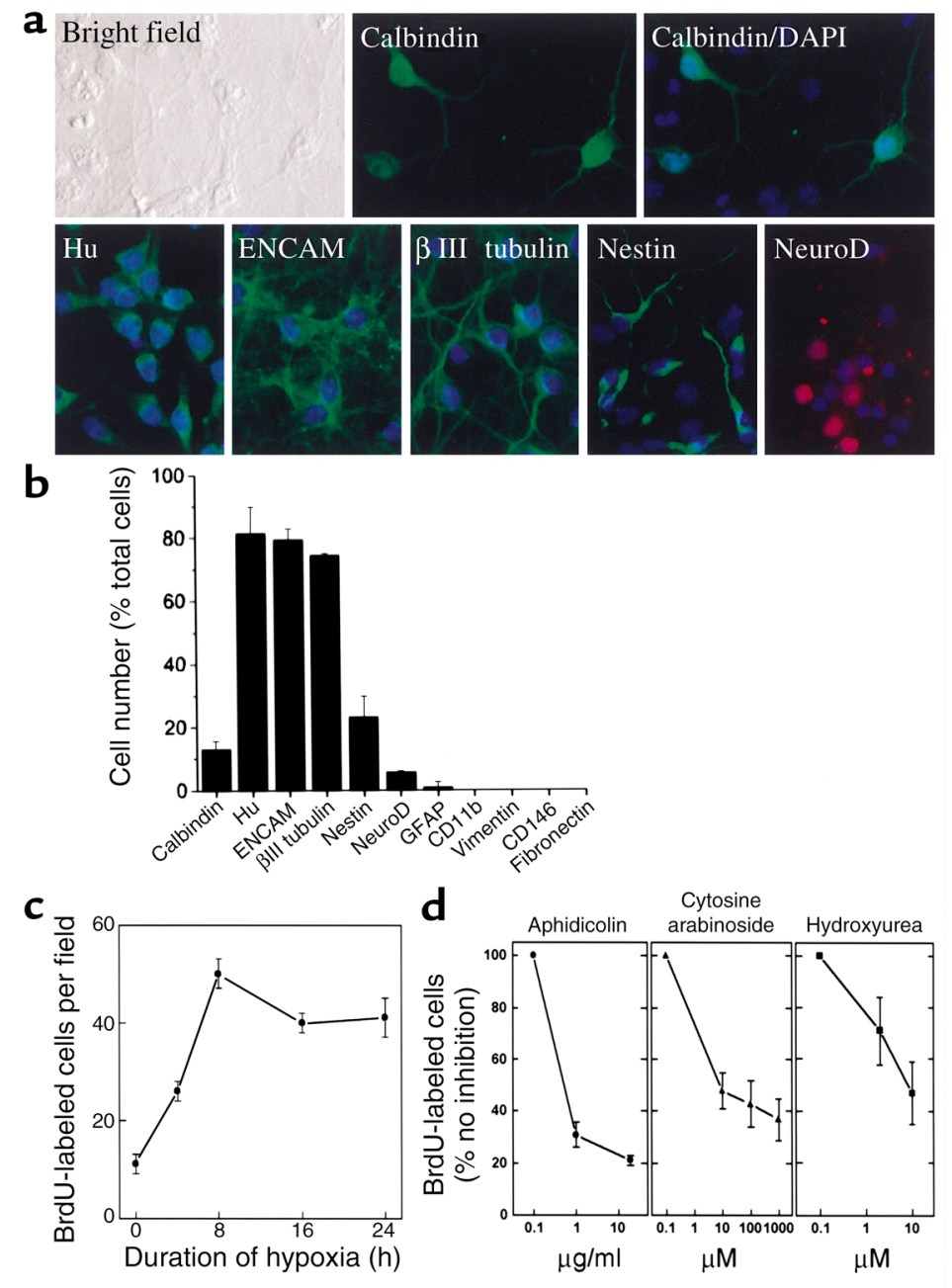




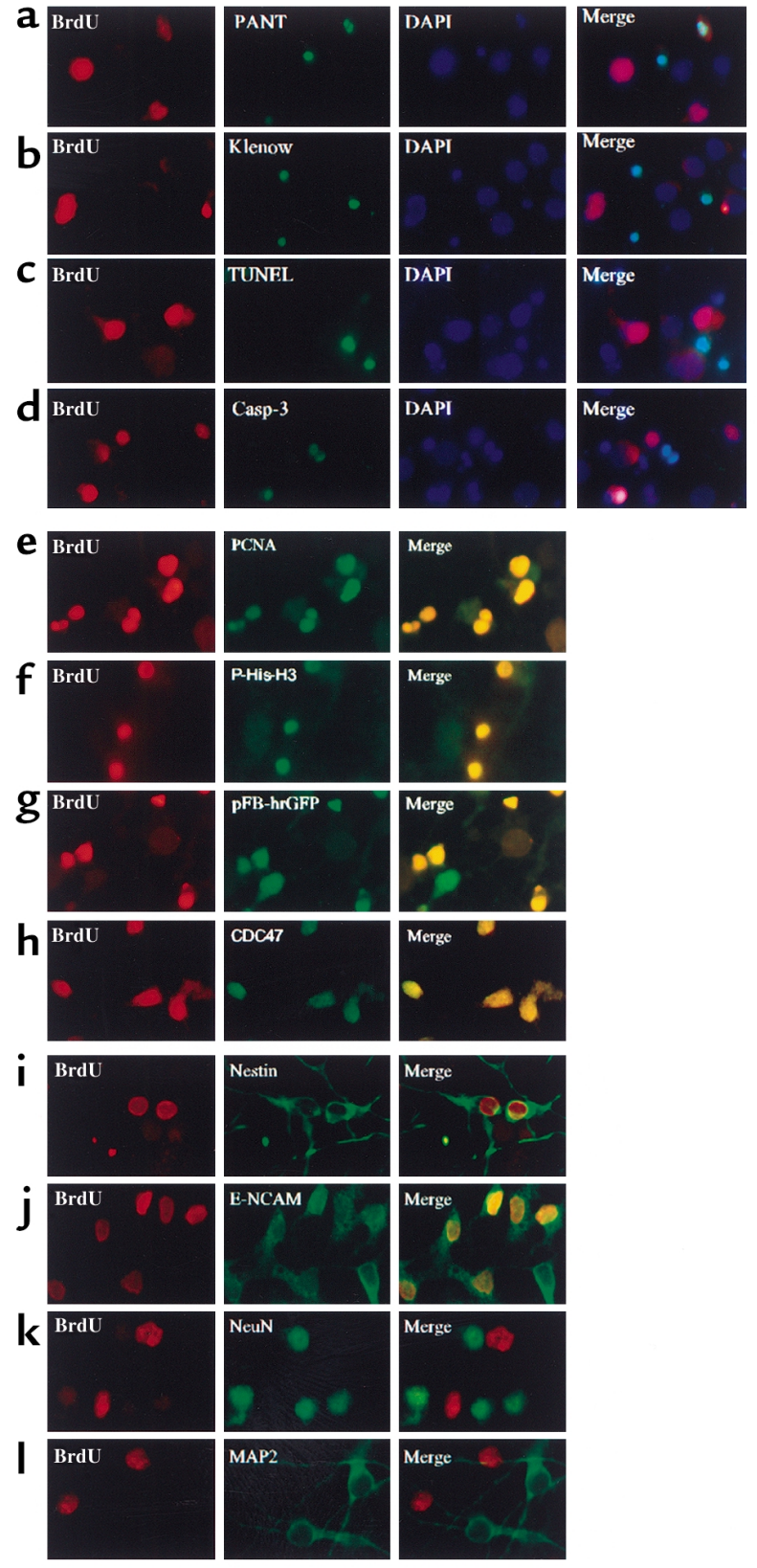

Figure 2

Hypoxia induces BrdU incorporation into uninjured cells that coexpress markers of proliferating cells and of immature, but not mature, neurons. Cerebral cortical cultures were treated with BrdU, exposed to hypoxia for 8 hours, and labeled with an Ab against BrdU and another marker, and nuclei were counterstained with DAPI. Most cells incorporating BrdU showed no evidence of DNA damage as assayed by PANT labeling (a), Klenow labeling (b), or TUNEL (c), or of caspase activation measured with an Ab against the 17- to $20-\mathrm{kDa}$ caspase- 3 cleavage product (d). BrdU incorporation colocalized with the cell proliferation markers PCNA (e) and phospho-histone- $\mathrm{H} 3(\mathbf{f})$, with retroviral infectivity reported by a GFP-expressing vector $(\mathbf{g})$, and with the "replication-licensing" protein CDC47 (h). As shown in $\mathbf{g}$, not all pFB-hrGFP-infected cells incorporated BrdU, which may be related to differences in labeling efficiency between the two markers. BrdU incorporation also colocalized with nestin (i) and to a large extent with E-NCAM (j), but not with the mature neuronal markers $\operatorname{NeuN}(\mathbf{k})$ and MAP-2 (I). Data are representative fields from at least three experiments per row.

\section{Results}

Hypoxia induces neurogenesis in neuronal cultures from mouse cerebral cortex. In previous studies of in vitro hypoxia we used cortical cultures maintained for 10-19 days, which contained greater than $90 \%$ mature neurons. To determine the cellular composition of 6- to 7-day cultures, immunocytochemistry was performed using cell type-specific markers. As shown in Figure 1, a and b, most cells expressed markers associated with immature neurons - Hu, E-NCAM, and $\beta I I I$ tubulin. Smaller percentages of cells expressed the neuroepithelial cell marker nestin, the mature neuronal marker calbindin, or the immature neuronal marker Neuro D. Some cells expressed both mature and immature neuronal markers, suggesting an intermediate stage of differentiation.

To determine if hypoxia stimulates BrdU incorporation, $\mathrm{BrdU}$ was added, and 20 minutes later cultures were placed in $95 \% \mathrm{~N}_{2} / 5 \% \mathrm{CO}_{2}$ for up to 24 hours. Under these conditions, the number of BrdU-labeled cells increased by 4 hours and was maximal $(\sim 5$ times control) at 8 hours (Figure 1c). To evaluate the possibility that BrdU labeling was due to DNA repair rather than replication, BrdU-labeled hypoxic cultures were assessed for DNA damage using the Klenow fragment of DNA polymerase I, PANT labeling, and TUNEL (31, 32 ). DNA damage was detectable in only approximately $20 \%$ of BrdU-labeled cells after 8 hours of hypoxia (Figure 2, a-c), and little overlap was seen between BrdU labeling and immunoreactivity for the 17- to 20$\mathrm{kDa}$ cleavage product of activated caspase-3 (Figure $2 \mathrm{~d}$ ), which is associated with hypoxic neuronal death in these cultures (33). To investigate further whether BrdU labeled primarily dividing cells in our cultures, hypoxia was induced in the presence of the cell cycle inhibitors aphidicolin, cytosine arabinoside, and hydroxyurea. Each cell cycle inhibitor reduced BrdU incorporation into hypoxic cultures by up to approximately $60-80 \%$ (Figure 1d), consistent with the majority of BrdU labeling being associated with cell division.

BrdU labeling also colocalized with several markers of cell proliferation, including PCNA (Figure 2e), a cell cycle-dependent nuclear protein that acts in concert with DNA polymerase- $\delta$ and helps to regulate the fidelity of DNA replication (34); phospho-histone-H3 (Figure 2f), a marker of mitotic chromosome condensation (35); a green fluorescent protein-expressing retroviral vector ( $\mathrm{pFB}-\mathrm{hrGFP}$ ) that infects dividing cells (Figure 2g); and the "replication-licensing" protein CDC47 (Figure 2h) (36).

To determine the identity of the BrdU-labeled cells, cultures were examined for coexpression of two markers expressed by neuroepithelial precursor cells or immature neurons, nestin (37) and E-NCAM (38), and two markers expressed by mature neurons, NeuN and MAP-2. Most BrdU-labeled cells coexpressed nestin and E-NCAM, but not NeuN or MAP-2 (Figure 2, i-l). This is additional evidence that $\mathrm{BrdU}$ incorporation is not simply due to DNA damage and repair, because DNA damage affects mature as well as immature neurons 
(39). BrdU labeling was also absent from the rare $(\sim 1 \%)$ GFAP-immunopositive cells in these cultures.

Hypoxia-induced neurogenesis is mediated by a soluble factor that can transfer neuroproliferative activity to normoxic cultures. Focal cerebral ischemia induces BrdU incorporation in neuroproliferative zones remote from the site of injury (20), suggesting that brain lesions may release diffusible factors that can stimulate neurogenesis. If such factors also mediate hypoxia-induced neurogenesis in our cultures, it should be possible to transfer neuroproliferative activity via hypoxia-conditioned medium (HCM). To test this possibility, cultures were exposed to hypoxia for up to 24 hours, and HCM was removed and transferred to normoxic cultures. BrdU was added, and cultures were maintained under normoxic conditions for up to 72 hours (Figure 3a). As predicted, HCM stimulated an increase in BrdU incorporation into normoxic cultures (Figure 3b). Like hypoxia-induced BrdU labeling, HCM-induced labeling colocalized with markers of cell proliferation and of immature but not mature neurons (Figure 3, d-f). Therefore, HCM can transfer the effect of hypoxia to normoxic cells.

Hypoxia-induced proliferative activity is associated with selected HCM fractions. To identify factors in HCM responsible for stimulating BrdU incorporation in our cultures, $\mathrm{HCM}$ was fractionated according to molecular weight by centrifugal filtration, and fractions were tested for their effects on cell viability and cell proliferation in normoxic cultures. Higher-molecular-weight (especially 30-50 $\mathrm{kDa})$ fractions stimulated BrdU incorporation and increased the number of dividing (pFB-hrGFP infectible) cells in normoxic cultures (Figure $3 \mathrm{~b}$ ). However, when HCM and HCM fractions were boiled and then added to cultures, there was no difference in $\mathrm{BrdU}$ incorporation across treatments $(P=0.148$, ANOVA).

Neither whole HCM nor 30-kDa or greater fractions reduced the number of viable cells in cultures measured by MTT absorbance, but the 10- to $30-\mathrm{kDa}$ fraction killed most cells by 72 hours and the $3-$ to $10-\mathrm{kDa}$ and less than $3-\mathrm{kDa}$ fractions killed almost all cells by 24 hours (Figure 3c). The toxic factors in these fractions do not appear to include excitatory amino acids because L-glutamate did not reduce cell viability and glutamate antagonists failed to inhibit the toxicity of 30-kDa or lower HCM fractions (not shown).

Candidate mediators of bypoxia-induced neurogenesis include SCF and FGF-2. Because maximal proliferative activity was associated with the $30-$ to $50-\mathrm{kDa}$ fraction of HCM, because many growth factors fall within this range, and because several of these have been implicated in neurogenesis, we screened a series of growth factors for increased expression in hypoxic neuronal cultures. These included EGF, FGF-2, BDNF, nerve growth factor, IGF-I, TNF- $\alpha$, TGF- $\alpha$, glial cell line-derived neurotrophic factor, and SCF. SCF and FGF-2 protein and mRNA levels were increased in hypoxic cultures (Figure $4, a, b$, and d). Elevated levels of SCF in HCM were also demonstrable by Western blotting of concentrated supernatants from centrifuged cultures (Figure 4c).
Because FGF-2 has been implicated previously in ischemia-induced neurogenesis (17), we focused our attention on SCF (also known as Steel factor, kit ligand, or mast cell growth factor), a secreted glycoprotein that migrates with an apparent molecular weight of 30-35 $\mathrm{kDa}(40)$. Mice with mutations in SCF or its receptor, c-kit, show amastocytosis, amelanocytosis, and sterility, providing evidence for the involvement of SCF in hematopoiesis, melanogenesis, and gametogenesis (41). SCF is also expressed in peripheral $(42,43)$ and central (44-46) neurons, has neurotrophic effects (47-49), and

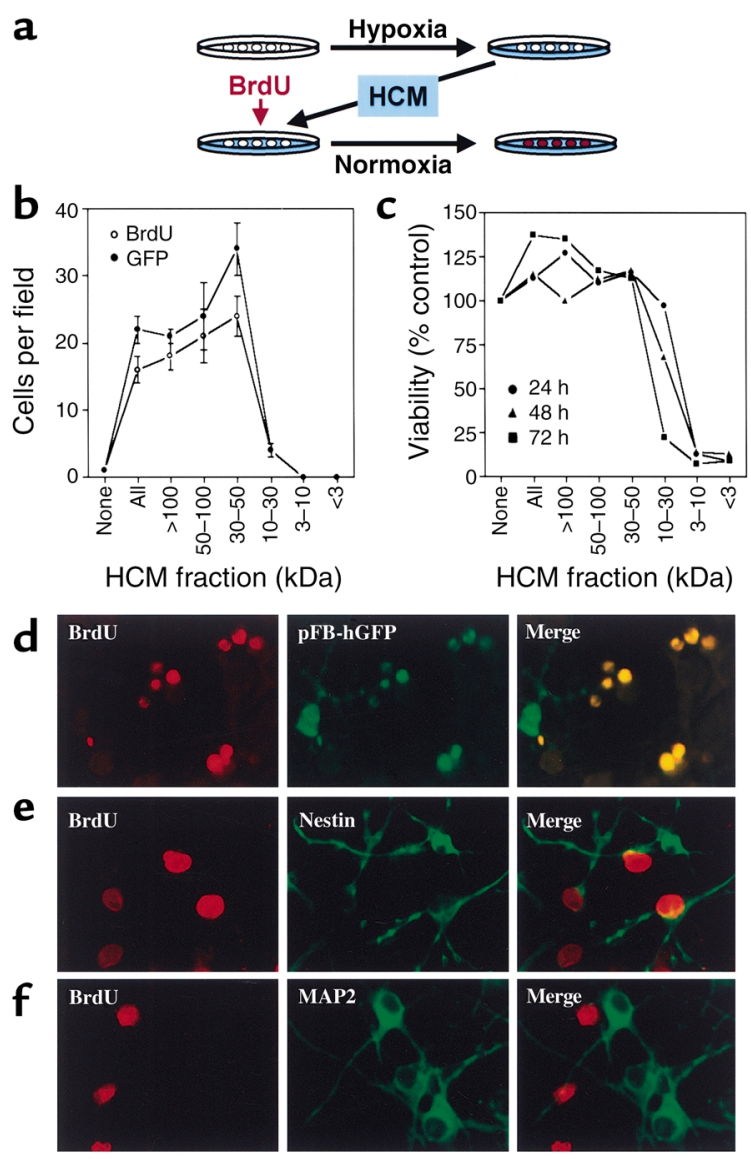

\section{Figure 3}

HCM transfers hypoxic stimulation of neurogenesis to normoxic cultures. Cerebral cortical cultures were exposed to normoxia or hypoxia for 8 hours, medium was removed, and normoxia-conditioned control medium (none), whole HCM (all), or HCM fractions were added to normoxic cultures, together with BrdU and, in some cases, pFB-hrGFP, for 24-72 hours (a). Incubation for 72 hours with HCM fractions of more than $30 \mathrm{kDa}$ increased the number of cells showing BrdU incorporation and GFP fluorescence (b). HCM fractions of less than $30 \mathrm{kDa}$ had no effect on BrdU or pFB-hrGFP labeling, but reduced cell viability at 24-72 hours as measured by MTT absorbance (c). To characterize BrdU-labeled cells, cultures were exposed to hypoxia for 8 hours, medium was removed, and HCM was added to normoxic cultures, together with BrdU, and in some cases, pFB-hrGFP (d) for 72 hours. Some cultures were also stained with Ab's against nestin (e) or MAP-2 (f). BrdU incorporation colocalized with pFB-hrGFP infectivity and with nestin, but not MAP-2 immunostaining. Data are mean \pm SEM, $n=3$ (b), mean values that varied by less than $10 \%, n=3$ (c), or representative fields from three experiments $(\mathbf{d}-\mathbf{f})$. 


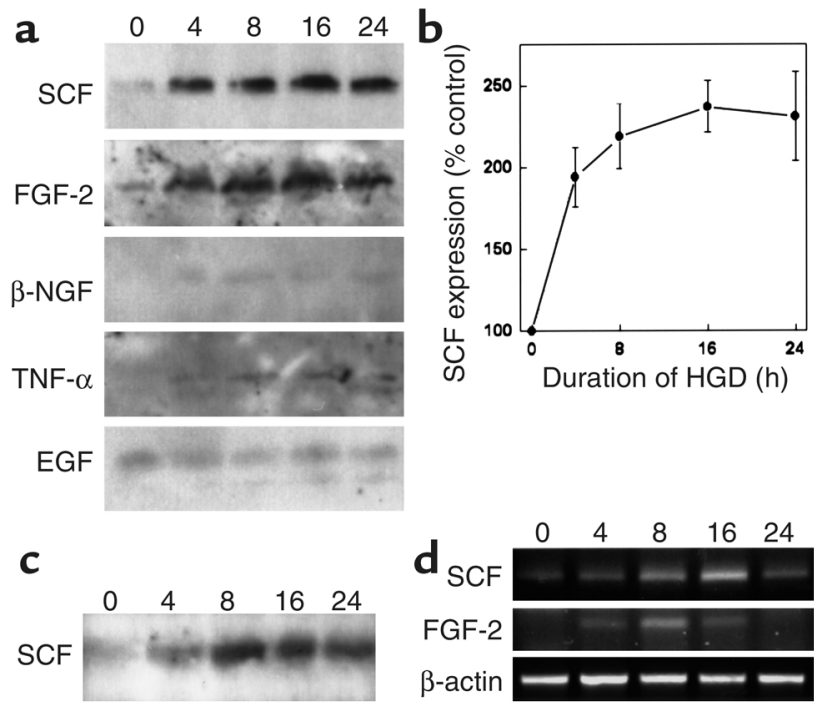

regulates neurosecretory function (50). Moreover, SCF mutations are associated with abnormal development of sensory neurons (51) and defects in hippocampusdependent learning (52). Finally, traumatic brain injury can increase neuronal SCF expression (53). However, SCF has not been implicated in neurogenesis.

If SCF contributes to the neuroproliferative effect of HCM, then a similar effect should be achieved by treating normoxic cultures with SCF alone. When SCF was added to cultures for 24 hours at concentrations comparable to those found in circulating blood and those associated with half-maximal effects of SCF in in vitro colony-forming assays $(2-9 \mathrm{ng} / \mathrm{ml})(40)$, we observed an increase of approximately $75 \%$ in the number of cells labeled with BrdU (Figure 5a). We also observed

\section{Figure 4}

Hypoxia induces expression of SCF and FGF-2 in vitro. Cerebral cortical cultures were exposed to hypoxia for up to 24 hours, and cellular expression of growth factors and cytokines was measured by Western blotting (a), which showed increased expression of SCF and FGF-2 at 4-24 hours. SCF expression was quantified by computer densitometry (b). SCF levels were also increased after the indicated periods of hypoxia (h) in culture supernatants (c). Enhanced expression of SCF and FGF-2 was due to transcriptional activation, because RT-PCR showed increases in SCF and FGF-2 mRNA levels (d). Data are representative blots from three experiments (a, c, d) or mean \pm SEM, $n=3$ (b).

an increase of approximately $40 \%$ in MTT absorbance, consistent with an increase in the number of viable cells in culture. FGF-2 also stimulated BrdU incorporation (Figure $5 \mathrm{~b}$ ), but the effects of maximally effective concentrations $(10 \mathrm{ng} / \mathrm{ml})$ of SCF and FGF-2 were not additive (Figure 5c). When HCM was treated with Ab's against SCF or FGF-2, stimulation of BrdU labeling was reduced by approximately $40 \%$, but the effects of the two Ab's were not additive (Figure 5d). Anti-SCF preferentially reduced BrdU labeling stimulated by the 30- to 50-kDa fraction of HCM, consistent with the $\mathrm{M}_{\mathrm{r}}$ value for SCF (30-35 kDa), whereas anti-FGF-2 was more effective in inhibiting the effect of the 10- to 30$\mathrm{kDa}$ fraction of HCM, consistent with the $\mathrm{M}_{\mathrm{r}}$ value for FGF-2 (17.2 kDa).

The effects of SCF, including its effects on neurons, are mediated through the c-kit receptor tyrosine kinase (54), and embryonic cortical neurons express c-kit in culture (46). To confirm that c-kit was expressed in our cultures, immunocytochemistry was performed with Ab's against SCF and against c-kit. In normoxic cultures, most cells expressed c-kit, while a more restricted population of cells expressed high levels of SCF (Fig- a

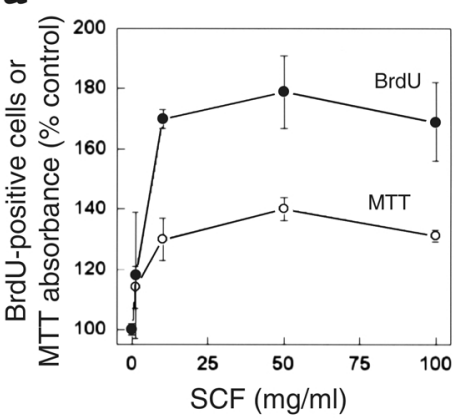

b

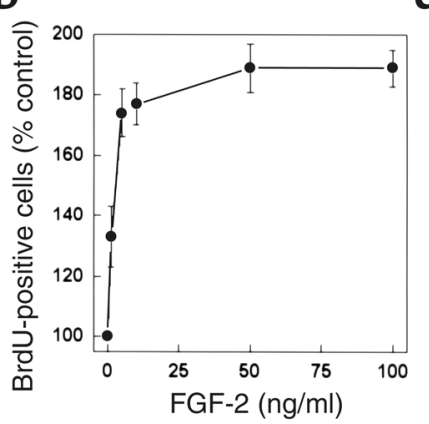

c

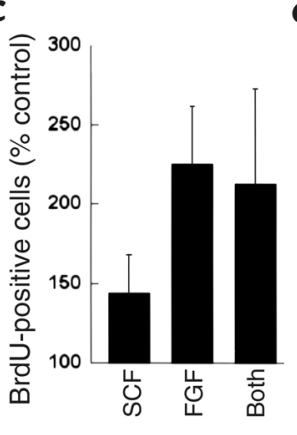

d

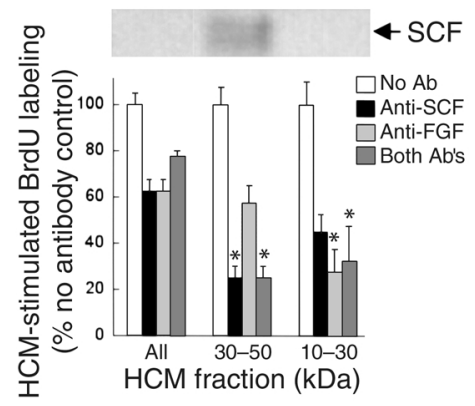

Figure 5

SCF and FGF-2 stimulate BrdU incorporation in vitro. Treatment of normoxic cultures for 24 hours with SCF increased both the number of cells incorporating BrdU and the number of viable cells measured by MTT absorbance (a). FGF-2 also increased BrdU incorporation in these cultures (b), but the effects of maximally effective concentrations $(10 \mathrm{ng} / \mathrm{ml})$ of SCF and FGF-2 were not additive (c). Ab's against SCF and FGF-2 each reduced the component of BrdU labeling stimulated by unfractionated HCM (all) by approximately $40 \%$, but these effects were not additive (d). Anti-SCF and the combination of anti-SCF plus anti-FGF-2 (but not anti-FGF-2 alone) reduced BrdU labeling stimulated by the 30 - to $50-\mathrm{kDa}$ fraction of $\mathrm{HCM}$ by approximately $75 \%$, consistent with the preferential localization of immunoreactive SCF to this fraction on Western blots. Anti-FGF-2 and the combination of anti-FGF-2 plus anti-SCF (but not anti-SCF alone) reduced BrdU labeling stimulated by the 10- to $30-\mathrm{kDa}$ fraction of $\mathrm{HCM}$ by approximately $70 \%$, consistent with the $\mathrm{M}_{\mathrm{r}}$ value of $17.2 \mathrm{kDa}$ for FGF-2. Data are mean $\pm \mathrm{SEM}, n=3$, or representative blots from three experiments (inset to $\mathbf{d}$ ). Asterisks in $\mathbf{d}$ indicate that the percentage of inhibition of BrdU labeling by a given Ab or combination of Ab's is significantly different in that HCM fraction than in unfractionated HCM $(P<0.05$; ANOVA and post hoc Student-Newman-Keuls tests). 


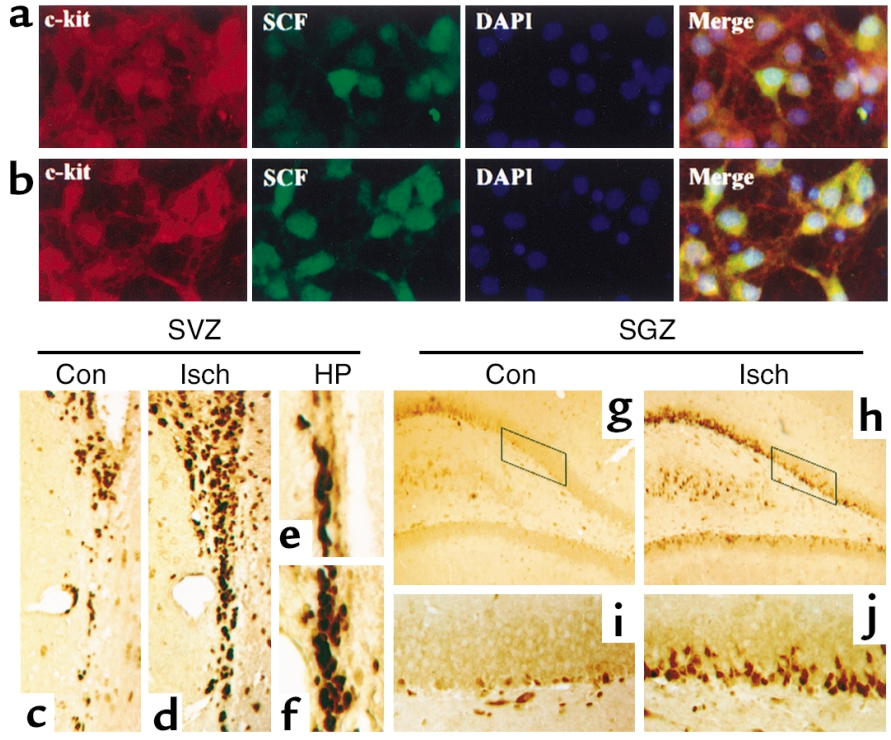

ure 6a). After 8 hours of hypoxia, however, most cells expressed both c-kit and SCF (Figure 6b).

SCF stimulates neurogenesis in vivo. To evaluate if SCF acts as a neuroproliferative factor in the brain in vivo, we first examined if $c$-kit is expressed in neuroproliferative zones. As shown in Figure 6, c-j, c-kit was expressed in both SVZ and SGZ. Moreover, the number of c-kit-expressing cells increased in the ipsilateral hemisphere after cerebral ischemia induced by MCA occlusion. The presence of c-kit in SVZ and SGZ and its upregulation after ischemia are compatible with the involvement of SCF in stimulation of neurogenesis after cerebral ischemia.

To test whether SCF can stimulate neurogenesis in vivo, adult rats were treated with SCF (infused into the right lateral ventricle) and $\mathrm{BrdU}$ (injected intraperitoneally) for 3 days, and sacrificed 1 week later. As shown in Figure 7,

\section{Figure 7}

Intraventricular SCF stimulates BrdU incorporation in SGZ and SVZ in vivo. (a) Brain sections through SGZ and SVZ were immunostained with anti-BrdU Ab 1 week after intraventricular infusion of SCF or aCSF vehicle $(n=6)$. Compared with aCSF (control), SCF increased the number of BrdU-positive cells in SGZ and SVZ. Proliferation was more pronounced on the cannula side, as compared with the contralateral side. (b) BrdU-labeled cells in SGZ (top) and SVZ (bottom) were counted in control brain $(n=6)$ and both ipsilateral (cannula side) and contralateral to SCF infusion $(n=6)$. In some experiments, SCF was infused together with anti-c-kit Ab $(n=4)$. Bars (left to right) represent control; SCF-treated, cannula side; SCF-treated, control side; and SCF- and anti-c-kit-treated, cannula side. BrdU-positive cells were increased in SVZ on both the infused and contralateral sides and in SGZ on the infused side. The effect of SCF was partially blocked by coadministration of anti-c-kit $A b\left({ }^{*} P<0.05\right.$, Student $t$ test). (c) Rat brain sections through SGZ, obtained 1 week after SCF infusion, were double-labeled for BrdU (red) and NeuN or Neuro D (green). Merged images show that BrdU labeling colocalized with Neuro D and, in some cases, NeuN. Data shown are representative fields from the number of experiments given above (a and $\mathbf{c}$ ), or mean $\pm \operatorname{SEM}(n=3)(\mathbf{b})$.

\section{Figure 6}

The c-kit expression in vitro and in SVZ and SGZ of normal and ischemic rat brain in vivo. Normoxic cerebral cortical cultures (a) and cultures deprived of oxygen for 8 hours (b) were stained with Ab's against c-kit and SCF and with DAPI. Brain sections through SVZ (c-f) and SGZ of DG $(\mathbf{g}-\mathbf{j})$ were also immunostained with an $A b$ against $c-k i t$, which was visualized with DAB. c-kit was expressed in SVZ (c and $\mathbf{e}$ ) and SGZ ( $\mathbf{g}$ and i) of normal brain (Con), and expression was increased 24 hours after MCA occlusion (Isch) in the ipsilateral SVZ (d and f) and SGZ (h and j). (c, d, g, and $\mathbf{h}) \times 200$. (e, f, i, and $\mathbf{j}) \times 400$ (HP). Data are representative fields from at least three experiments per panel.

$a$ and $b$, the number of BrdU-immunopositive cells in SVZ and SGZ increased after SCF administration, especially ipsilateral to the infusion. The increase in BrdU labeling was abolished by coadministration of an anti-c-kit Ab, suggesting that the effect of SCF was mediated through c-kit.

To identify cells targeted to proliferate by SCF, sections from SGZ of SCF- and BrdU-treated rat brains 1 week after treatment were immunostained for BrdU and for markers of mature $(\mathrm{NeuN})$ and immature (Neuro D) neurons (Figure 7c). These double-label studies showed that most BrdU-immunopositive cells coexpressed Neuro D and a few cells expressed NeuN. Therefore, SCF appears to stimulate proliferation of cells of neuronal lineage in vivo, most of which exhibit an immature neuronal phenotype at 1 week.
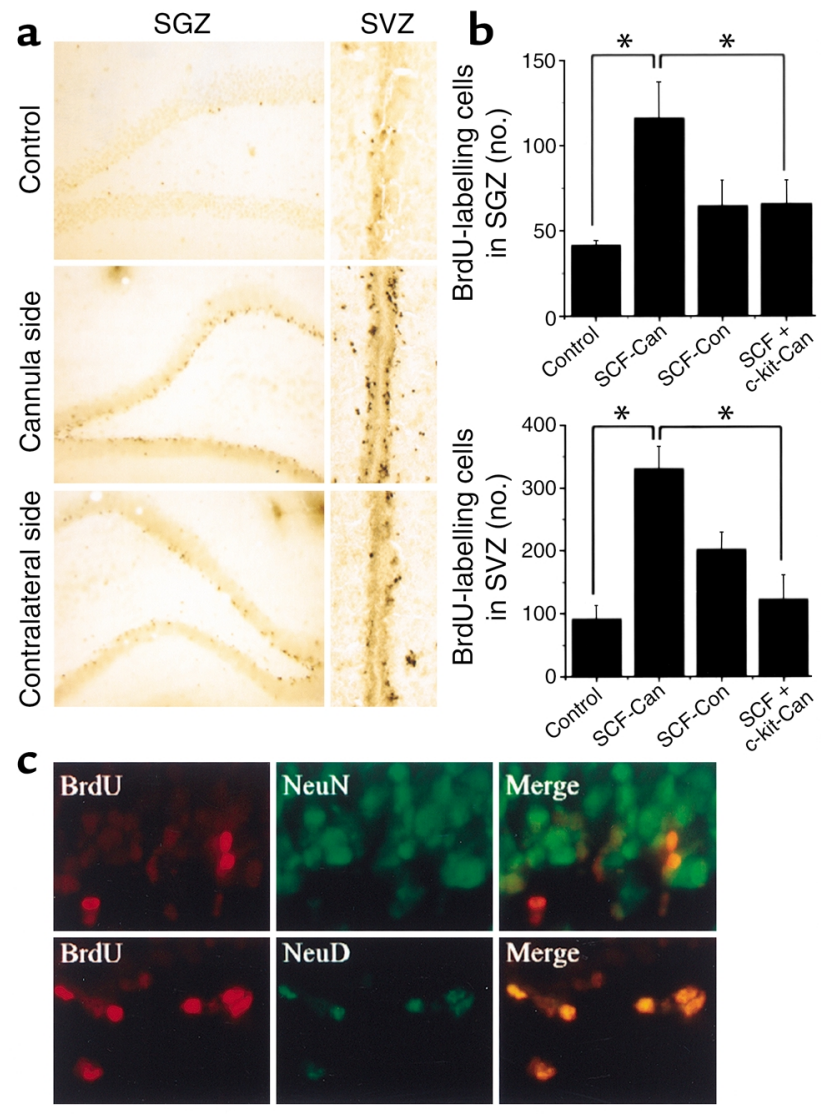


\section{Discussion}

Cerebral ischemia stimulates neurogenesis both locally (21-24) and at a distance $(17,20,55)$ and may even have the capacity to direct the fate of hematopoietic stem cells toward a neuronal phenotype (56). One way such lesion-derived signaling might occur is through the release of trophic factors from injured tissue.

To begin to identify factors that might contribute to ischemia-induced neurogenesis, we modeled cerebral ischemia in neuronal cultures from embryonic mouse cerebral cortex. Hypoxia stimulated the incorporation of BrdU into cells that coexpressed phenotypic markers of proliferating cells and immature neurons, suggesting that they are dividing cells that might have the capacity to develop into mature, functional neurons. Next we transferred proliferative activity to normoxic cultures with HCM. Its effect was similar to that of hypoxia and was associated preferentially with HCM constituents of $30 \mathrm{kDa}$ or greater. Among several possible mediators tested, SCF and FGF-2 were both upregulated in hypoxia-treated cultures, and both increased BrdU incorporation. Although synergistic effects of SCF and FGF-2 have been described in myeloid progenitor cells (57), the effects of SCF and FGF-2 were not additive in our cultures, which could imply that they act through a shared signal transduction pathway. Alternatively, one of these growth factors could act through effects on the other: for example, SCF increases FGF-2 expression in mast cells (58) and FGF-2 increases SCF expression in several murine cell lines (59).

SCF produces biological effects by activating c-kit, leading to receptor homodimerization and autophosphorylation and stimulating signal transduction pathways involving phosphatidylinositol-3-kinase, Src, JAK/STAT, and Ras-Raf-MAP kinase (54). In hematopoietic cells, SCF and c-kit regulate a variety of developmental events, including cell proliferation, survival, and differentiation (60). An effect of SCF alone is insufficient to explain the neuroproliferative effects of hypoxia and HCM in our cultures, because the maximal percentage increase in BrdU incorporation observed with SCF was less than that seen with hypoxia or HCM and because neuroproliferative effects were associated with several HCM fractions and with FGF-2.

Several pitfalls can beset studies of this sort (39). First, BrdU can label cells undergoing DNA repair, but we did not find evidence of DNA damage by PANT labeling, Klenow labeling, or TUNEL in most BrdUlabeled cells. In addition, BrdU labeling colocalized with other markers of cell proliferation, including PCNA, phospho-histone-H3, and CDC47 (36). Finally, BrdU immunoreactivity correlated with retroviral infectivity. Another issue is whether proliferating cells that express neuronal markers are necessarily destined to become neurons or simply exhibit ectopic expression of such markers as part of an injury response. The BrdU-labeled cells we studied in vitro expressed both nestin and E-NCAM, which suggests that they were neuroepithelial precursors or immature neurons. Whether they eventually would have expressed mature neuronal markers like NeuN and MAP-2, or electrophysiological neuronal properties if exposed to the proper combination and sequence of instructive signals in vivo, or achieve functional integration into an ischemic brain remains to be determined.

To address whether SCF stimulates neurogenesis in vivo, we examined whether (a) c-kit is expressed in neuroproliferative zones of the brain, (b) c-kit expression in SVZ and SGZ is altered after ischemia, (c) administration of SCF increases BrdU incorporation in SVZ and SGZ, and (d) cells in which SCF stimulates BrdU incorporation are of neuronal lineage. In all four cases, our results were affirmative, supporting a role for SCF in ischemia-induced neurogenesis in vivo.

The ability of an ischemic brain lesion to stimulate the proliferation of neuronal precursors invites speculation as to the possible role of such proliferation in functional recovery from stroke and the possibility that augmentation of neurogenesis, perhaps by the administration of one or more trophic factors, might have therapeutic potential. These issues are important because, although some degree of spontaneous recovery after stroke is the norm, the molecular and cellular mechanisms underlying recovery are poorly understood and existing treatment for stroke is generally applicable to only a small percentage of cases, or is limited in efficacy. Endogenous mechanisms of neuroprotection from ischemia, including mechanisms of neurogenesis, may hold clues for the development of improved therapy.

\section{Acknowledgments}

Supported by United States Public Health Service grant NS35965.

1. Kirschenbaum, B., and Goldman, S.A. 1995. Brain-derived neurotrophic factor promotes the survival of neurons arising from the adult rat forebrain subependymal zone. Proc. Natl. Acad. Sci. USA. 92:210-214.

2. Lois, C., and Alvarez-Buylla, A. 1993. Proliferating subventricular zone cells in the adult mammalian forebrain can differentiate into neurons and glia. Proc. Natl. Acad. Sci. USA. 90:2074-2077.

3. Luskin, M.B. 1993. Restricted proliferation and migration of postnatally generated neurons derived from the forebrain subventricular zone. Neuron. 11:173-189.

4. Altman, J. 1963. Autoradiographic study of cell proliferation in the brains of rats and cats. Anat. Rec. 145:573-591.

5. Rakic, P. 2002. Neurogenesis in adult primate neocortex: an evaluation of the evidence. Nature Rev. Neurosci. 3:65-71.

6. Reynolds, B.A., and Weiss, S. 1992. Generation of neurons and astrocytes from isolated cells of the adult mammalian central nervous system. Science. 255:1707-1710.

7. Ray, J., Peterson, D.A., Schinstine, M., and Gage, F.H. 1993. Proliferation, differentiation, and long-term culture of primary hippocampal neurons. Proc. Natl. Acad. Sci. USA. 90:3602-3606.

8. Kilpatrick, T.J., and Bartlett, P.F. 1993. Cloning and growth of multipotential neural precursors: requirements for proliferation and differentiation. Neuron. 10:255-265.

9. Taupin, P., et al. 2000. FGF-2-responsive neural stem cell proliferation requires CCg, a novel autocrine/paracrine cofactor. Neuron. 28:385-397.

10. Temple, S. 1989. Division and differentiation of isolated CNS blast cells in microculture. Nature. 340:471-473.

11. Dehay, C., Savatier, P., Cortay, V., and Kennedy, H. 2001. Cell-cycle kinetics of neocortical precursors are influenced by embryonic thalamic axons. J. Neurosci. 21:201-214.

12. Benraiss, A., Chmielnicki, E., Lerner, K., Roh, D., and Goldman, S.A. 2001. Adenoviral brain-derived neurotrophic factor induces both neos- 
triatal and olfactory neuronal recruitment from endogenous progenitor cells in the adult forebrain. J. Neurosci. 21:6718-6731.

13. Pencea, V., Bingaman, K.D., Wiegand, S.J., and Luskin, M.B. 2001. Infusion of brain-derived neurotrophic factor into the lateral ventricle of the adult rat leads to new neurons in the parenchyma of the striatum, septum, thalamus, and hypothalamus. J. Neurosci. 21:6706-6717.

14. Wagner, J.P., Black, I.B., and DiCicco-Bloom, E. 1999. Stimulation of neonatal and adult brain neurogenesis by subcutaneous injection of basic fibroblast growth factor. J. Neurosci. 19:6006-6016.

15. Zigova, T., Pencea, V., Wiegand, S.J., and Luskin, M.B. 1998. Intraventricular administration of BDNF increases the number of newly generated neurons in the adult olfactory bulb. Mol. Cell Neurosci. 11:234-245.

16. Gould, E., and Tanapat, P. 1997. Lesion-induced proliferation of neuronal progenitors in the dentate gyrus of the adult rat. Neuroscience. 80:427-436.

17. Yoshimura, S., et al. 2001. FGF-2 regulation of neurogenesis in adult hippocampus after brain injury. Proc. Natl. Acad. Sci. USA. 98:5874-5879.

18. Parent, J.M., et al. 1997. Dentate granule cell neurogenesis is increased by seizures and contributes to aberrant network reorganization in the adult rat hippocampus. J. Neurosci. 17:3727-3738.

19. Magavi, S.S., Leavitt, B.R., and Macklis, J.D. 2000. Induction of neurogenesis in the neocortex of adult mice. Nature, 405:951-955.

20. Jin, K., et al. 2001. Neurogenesis in dentate subgranular zone and rostra subventricular zone after focal cerebral ischemia in the rat. Proc. Natl. Acad. Sci. USA. 98:4710-4715.

21. Liu, J., Solway, K., Messing, R.O., and Sharp, F.R. 1998. Increased neurogenesis in the dentate gyrus after transient global ischemia in gerbils. J. Neurosci. 18:7768-7778.

22. Takagi, Y., et al. 1999. Proliferation of neuronal precursor cells in the dentate gyrus is accelerated after transient forebrain ischemia in mice. Brain Res. 831:283-287.

23. Gu, W., Brannstrom, T., and Wester, P. 2000. Cortical neurogenesis in adult rats after reversible photothrombotic stroke. J. Cereb. Blood Flow Metab. 20:1166-1173.

24. Jiang, W., Gu, W., Brännström, T., Rosqvist, R., and Wester, P. 2001. Cortical neurogenesis in adult rats after transient middle cerebral artery occlusion. Stroke. 32:1201-1207.

25. Koretz, B., Ahern, K.v.B., Lustig, H.S., and Greenberg, D.A. 1994. Pre- and postsynaptic modulators of excitatory neurotransmission: comparative effects on hypoxia/hypoglycemia in cortical cultures. Brain Res. 643:334-337.

26. Bayer, S.A., and Altman, J. 1995. Neurogenesis and neuronal migration. In The rat nervous system. G. Paxinos, editor. Academic Press. San Diego, California, USA. 1041-1078.

27. Jin, K.L., Mao, X.O., and Greenberg, D.A. 2000. Vascular endothelial growth factor: direct neuroprotective effect in in vitro ischemia. Proc. Natl. Acad. Sci. USA. 97:10242-10247.

28. Brewer, G.J., Torricelli, J.R., Evege, E.K., and Price, P.J. 1993. Optimized survival of hippocampal neurons in B27-supplemented Neurobasal, a new serum-free medium combination. J. Neurosci. Res. 35:567-576.

29. Pulsinelli, W.A., Brierley, J.B., and Plum, F. 1982. Temporal profile of neuronal damage in a model of transient forebrain ischemia. Ann. Neurol. 11:491-498.

30. Sun, Y., Jin, K., Mao, X.O., Zhu, Y., and Greenberg, D.A. 2001. Neuroglobin is upregulated by and protects neurons from hypoxic-ischemic injury. Proc. Natl. Acad. Sci. USA. 98:15306-15311.

31. Chen, J., et al. 1997. Early detection of DNA strand breaks in the brain after transient focal ischemia: implications for the role of DNA damage in apoptosis and neuronal cell death. J. Neurochem. 69:232-245.

32. Jin, K., et al. 1999. In situ detection of neuronal DNA strand breaks using the Klenow fragment of DNA polymerase I reveals different mechanisms of neuron death after global cerebral ischemia. J. Neurochem. 72:1204-1214

33. Jin, K., et al. 2001. Caspase-3 and the regulation of hypoxic neuronal death by vascular endothelial growth factor. Neuroscience. 108:351-358.

34. Ino, H., and Chiba, T. 2000. Expression of proliferating cell nuclear antigen (PCNA) in the adult and developing mouse nervous system. Brain Res. Mol. Brain Res. 78:163-174.

35. Hendzel, M.J., et al. 1997. Mitosis-specific phosphorylation of histone $\mathrm{H} 3$ initiates primarily within pericentromeric heterochromatin during G2 and spreads in an ordered fashion coincident with mitotic chromo- some condensation. Chromosoma. 106:348-360.

36. Fujita, M., Kiyono, T., Hayashi, Y., and Ishibashi, M. 1996. hCDC47, a human member of the MCM family. Dissociation of the nucleus-bound form during S phase. J. Biol. Chem. 271:4349-4354.

37. Cattaneo, E., and McKay, R. 1990. Proliferation and differentiation of neuronal stem cells regulated by nerve growth factor. Nature. 347:762-765.

38. Seki, T., and Arai, Y. 1991. The persistent expression of a highly polysialylated NCAM in the dentate gyrus of the adult rat. Neurosci. Res. 12:503-513.

39. Scharff, C. 2000. Chasing fate and function of new neurons in adult brains. Curr. Opin. Neurobiol. 10:774-783.

40. Molineux, G., and McNiece, I.K. 1998. Stem cell factor. In The cytokine bandbook. A.W. Thomson, editor. Academic Press. San Diego, California, USA. 713-725.

41. Galli, S.J. 1993. New concepts about the mast cell. N. Engl. J. Med. 328:257-265.

42. Torihashi, S., Yoshida, H., Nishikawa, S., Kunisada, T., and Sanders, K.M. 1996. Enteric neurons express Steel factor-lacZ transgene in the murine gastrointestinal tract. Brain Res. 738:323-328.

43. Young, H.M., Torihashi, S., Ciampoli, D., and Sanders, K.M. 1998. Identification of neurons that express stem cell factor in the mouse small intestine. Gastroenterology. 115:898-908.

44. Manova, K., et al. 1992. c-kit receptor and ligand expression in postnatal development of the mouse cerebellum suggests a function for c-kit in inhibitory interneurons. J. Neurosci. 12:4663-4676.

45. Wong, M.L., and Licinio, J. 1994. Localization of stem cell factor mRNA in adult rat hippocampus. Neuroimmunomodulation. 1:181-187.

46. Zhang, S.C., and Fedoroff, S. 1997. Cellular localization of stem cell factor and c-kit receptor in the mouse nervous system. J. Neurosci. Res. 47:1-15.

47. Carnahan, J.F., Patel, D.R., and Miller, J.A. 1994. Stem cell factor is a neurotrophic factor for neural crest-derived chick sensory neurons. J. Neurosci. 14:1433-1440.

48. Hirata, T., et al. 1993. Stem cell factor induces outgrowth of c-kit-positive neurites and supports the survival of c-kit-positive neurons in dorsal root ganglia of mouse embryos. Development. 119:49-56.

49. Langtimm-Sedlak, C.J., Schroeder, B., Saskowski, J.L., Carnahan, J.F., and Sieber-Blum, M. 1996. Multiple actions of stem cell factor in neural crest cell differentiation in vitro. Dev. Biol. 174:345-359.

50. Kovacs, K.J., Foldes, A., and Vizi, E.S. 1996. C-kit ligand (stem cell factor) affects neuronal activity, stimulates pituitary-adrenal axis and prolactin secretion in rats. J. Neuroimmunol. 65:133-141.

51. Lourenssen, S., Motro, B., Bernstein, A., and Diamond, J. 2000. Defects in sensory nerve numbers and growth in mutant Kit and Steel mice. Neuroreport. 11:1159-1165.

52. Motro, B., Wojtowicz, J.M., Bernstein, A., and van der Kooy, D. 1996. Steel mutant mice are deficient in hippocampal learning but not long-term potentiation. Proc. Natl. Acad. Sci. USA. 93:1808-1813.

53. Zhang, S.C., and Fedoroff, S. 1999. Expression of stem cell factor and c-kit receptor in neural cells after brain injury. Acta Neuropathol. (Berl.) 97:393-398.

54. Linnekin, D. 1999. Early signaling pathways activated by c-Kit in hematopoietic cells. Int. J. Biochem. Cell Biol. 31:1053-1074.

55. Zhang, R.L., Zhang, Z.G., Zhang, L., and Chopp, M. 2001. Proliferation and differentiation of progenitor cells in the cortex and the subventricular zone in the adult rat after focal cerebral ischemia. Neuroscience. 105:33-41.

56. Chen, J., et al. 2001. Therapeutic benefit of intravenous administration of bone marrow stromal cells after cerebral ischemia in rats. Stroke. 32:1005-1011.

57. Gabrilove, J.L., White, K., Rahman, Z., and Wilson, E.L. 1994. Stem cell factor and basic fibroblast growth factor are synergistic in augmenting committed myeloid progenitor cell growth. Blood. 83:907-910.

58. Qu, Z., et al. 1998. Synthesis of basic fibroblast growth factor by murine mast cells. Regulation by transforming growth factor beta, tumor necrosis factor alpha, and stem cell factor. Int. Arch. Allergy Immunol. 115:47-54.

59. Sugimoto, Y., Koji, T., and Miyoshi, S. 1999. Modification of expression of stem cell factor by various cytokines. J. Cell Physiol. 181:285-294.

60. Ashman, L.K. 1999. The biology of stem cell factor and its receptor c-kit. Int. J. Biochem. Cell Biol. 31:1037-1051. 